\title{
Le paysage intellectuel du développement durable en Amérique latine. Une analyse de réseau à partir des citations d'auteurs latino-américains
}

\author{
Julien Vanhulst ${ }^{1, *}$ et Edwin Zaccai ${ }^{2}$ \\ ${ }^{1}$ Sociologie, Universidad Católica del Maule, Faculté de sciences sociales et économiques, Talca, Chili \\ 2 Philosophie, sciences de l'environnement, Université libre de Bruxelles, IGEAT, Bruxelles, Belgique
}

Reçu le 29 mai 2017. Accepté le 8 novembre 2018

Dans un article publié dans NSS (1994, 2, 4), qui est devenu depuis une référence, Olivier Godard présentait un paysage intellectuel du développement durable. C'est ce à quoi s'attachent aussi les auteurs de cet article, en adoptant une approche et en mobilisant une méthodologie tout à fait différentes. Ils s'intéressent, en effet, à la manière dont cette notion - ô combien plastique ! - s'est diffusée et a été appropriée par la sphère académique latino-américaine entre 1970 et 2012 . Ils montrent ainsi comment elle s'est inscrite dans un paysage intellectuel particulier et l'a modelé, un paysage dans lequel, sur le plan conceptuel, se reflètent des débats sur les relations de domination entre le centre et la périphérie du capitalisme, le multiculturalisme et l'indigénisme. D’une certaine manière, cette perspective historique et sociopolitique fait écho à «l'anatomie » de la notion de développement durable à laquelle s'était livré Dominique Pestre dans un autre texte publié dans NSS (2011, 19, 1).

La Rédaction

\begin{abstract}
Résumé - Les discours scientifiques sont influencés par des visions du monde et des valeurs partagées par des communautés scientifiques. En matière de développement durable, comme pour d'autres sujets, la production, diffusion et réception de savoirs structurent l'univers discursif d'espaces académiques. Cet article propose une cartographie du champ des interactions académiques d'un échantillon de 93 intellectuels influents en matière de développement durable en Amérique latine à partir d'un examen de plus de dix mille citations sur une période de quarante ans (1972-2012). Cette analyse quantitative est complétée par une analyse qualitative portant sur le contenu de la pensée des auteurs les plus importants. Il en ressort une structuration progressive du champ discursif sur le sous-continent. Sur le fond, nous observons que beaucoup de discours académiques, construits en synergie avec certains mouvements sociaux qui luttent pour la justice et la soutenabilité socioenvironnementale, s'avèrent critiques.
\end{abstract}

Mots-clés : Amérique latine / réseau de citations / développement durable / discours académiques / analyse historique

\begin{abstract}
The intellectual landscape of sustainable development in Latin America. A network analysis based on citations of Latin-American authors. Scientific discourses are influenced by worldviews and values shared by scientific communities. In terms of sustainable development, as for other subjects, the production, dissemination and reception of knowledge structure the discursive universe of academic activities. This paper provides a mapping of the field of academic interactions based on a sample of 93 influential intellectuals active in sustainable development thinking in Latin America, from a review of more than ten thousand citations over a period of forty years (1972-2012). The software Gephi was used to draw figures that highlight these networks along three separate periods of time. A first period runs from 1970 and the start of modern environmental concerns until 1987 and the publication of the very influential Brundtland Report. A second period (1987-2002) covers the period of a flourishing institutional concept of sustainable development until the Johannesburg Conference. And the third one runs until the
\end{abstract}

\footnotetext{
*Auteur correspondant : julien@ucm.cl
} 
Rio+20 Summit in 2012. This quantitative analysis is complemented by a qualitative analysis of the works and relations of the most influential authors. The results show a gradual structuring of the discursive field on the subcontinent. We are able to trace the main relations between authors in Latin America and outside, mostly to the US and Europe. Our results also show that many academics in the sub region elaborated critical discourses that developed in synergy with various social movements that strive for justice and socioenvironmental sustainability.

Keywords: Latin America / citation network / sustainable development / academic discourse / historical analysis

\section{Le champ des discours du développement durable et son étude}

Depuis les dernières décennies du siècle passé, les débats portant sur la durabilité ont fait émerger de multiples propositions que nous regroupons ici sous l'expression de «discours du développement durable ${ }^{1}$. En effet, cette notion a été un catalyseur important du débat socioenvironnemental, devenant un référentiel central tant dans les politiques publiques que dans les répertoires de l'action collective de la société civile, les stratégies d'entreprises, et la recherche fondamentale et appliquée dans le domaine des sciences naturelles et humaines (Adams, 2001; Zaccai, 2002; Dryzek, 2005 ; Vivien, 2005). Toutefois, ce référentiel est loin d'avoir une signification univoque et renvoie plutôt à un large éventail d'interprétations fondées sur des visions du monde spécifiques (Lélé, 1991; 2013; Sneddon et al., 2006 ; Villalba, 2009; Van Opstal et Hugé, 2013), c'est pourquoi il a parfois été qualifié de «concept contestable» (Jacobs, 1999; Connelly, 2007). Ainsi, selon Jacobs $(1999$, p. 26), «il y a une lutte pour le sens du développement durable. Mais il est inutile d'essayer d'obtenir un "accord" universel sur un sens unitaire du terme. Cela n'arrivera jamais, étant donné que ceux qui l'utilisent ont des intérêts et des valeurs politiques différents. [...] Cela ne devrait pas être perçu comme un

\footnotetext{
${ }^{1} \mathrm{Ce}$ travail se fonde sur les résultats obtenus par Julien Vanhulst dans sa thèse de doctorat Les chemins sinueux $d u$ développement durable (Université libre de Bruxelles/Universidad Alberto Hurtado) soutenue en 2015 sous la direction d'Edwin Zaccai et d'Alejandro Pelfini. L'article s'inscrit dans le projet de recherche FONDECYT n 11180256 (équivalent ANR jeune chercheur) financé par la Commission nationale de recherche scientifique et technologique [CONICYT], Chili). Nous remercions Fanny Lajarthe pour son aide précieuse de traduction, Elena Pisani pour sa révision des instruments méthodologiques et des analyses de réseaux ainsi que les relecteurs anonymes pour leurs commentaires sur notre manuscrit original. Une première version en anglais de cet article a été publiée en 2016 dans la revue Environmental Development sous le titre «Sustainability in Latin America: An analysis of the academic discursive fiel» (Vanhulst et Zaccai, 2016).
}

manque de précision sur ce que le développement durable "veut dire": au contraire, cette contestation constitue la lutte politique pour le sens donné au développement social et économique ${ }^{2} »$.

Par conséquent, plutôt que de faciliter l'émergence d'un concept clairement délimité, l'idée de développement durable a contribué à la formation d'un champ discursif façonné par diverses appropriations, caractérisées non seulement par des hypothèses divergentes sur les causes et la nature du problème de la durabilité, mais également par des propositions différentes pour $\mathrm{y}$ répondre. Cette idée de «champ discursif du développement durable» a été introduite notamment par John Dryzek (2005) qui conteste l'idée d'un mouvement historique de convergence vers un «âge de la raison environnementale» et considère la problématique environnementale comme un espace de « disputes continuelles » entre acteurs porteurs de visions très différentes. Pour donner du sens à ces perspectives variées, il propose de partir de la notion de discours en tant que «manière partagée d'appréhender le monde» qui permet à ceux qui y adhèrent d'interpréter les informations et de les assembler dans un récit cohérent. Dryzek élabore pour ce faire une taxonomie de discours environnementaux selon deux dimensions : premièrement le degré de radicalité dans la critique de l'«industrialisme» (le discours dominant de la société industrielle). La seconde dimension caractérise le mode de redéfinition d'un scénario politique et économique plus ou moins transformateur. $\mathrm{Au}$ total, ces deux dimensions croisées produisent quatre catégories illustrées par huit discours (Dryzek, 2005, p. 14-15). Wolfgang Sachs (1997, p. 71) aborde également le développement durable comme un «champ discursif» et différencie les discours selon leur mode d'évaluation du développement et leur façon de lier écologie et justice sociale (Sachs W., 1997; 1999).

Pour leur part, Hopwood et al. (2005) ont proposé une typologie des discours du développement durable fondée sur deux axes principaux: 1) la dichotomie entre anthropocentrisme et écocentrisme ; 2) la tension entre égalités et inégalités. Cette classification met en

\footnotetext{
${ }^{2}$ Les citations dans une autre langue que le français ont été traduites par les auteurs.
} 
évidence que le champ discursif du développement durable se compose non seulement d'un pilier environnemental mais également d'un pilier relatif à la justice sociale. Ce même travail distingue trois manières de remettre en question les dispositions institutionnelles existantes dans le but d'assurer un développement durable: 1) le statu quo; 2) la réforme; 3) la transformation. Pour les partisans du statu quo, les réponses à l'enjeu central de la durabilité peuvent être formulées au sein des structures existantes (et donc aucun changement n'est requis à cet égard). Les discours réformistes, quant à eux, soulignent la nécessité de changements institutionnels graduels, sans toutefois promouvoir une réelle transformation. Enfin, le troisième courant appelle à une transformation radicale dans la mesure où il considère que le cœur du problème se situe dans les structures économiques et de pouvoir ainsi que dans les modes d'interaction contemporains entre les hommes et leur environnement.

Nous utiliserons ces trois catégories de Hopwood et al. (2005) dans notre analyse pour caractériser les modes d'appropriation des discours du développement durable, et en particulier les deux dernières, que nous avons identifiées comme dominant les discours intellectuels autour du développement durable en Amérique latine. Nous partons du postulat que les discours scientifiques ne sont pas neutres et qu'ils répondent aux visions du monde et aux valeurs partagées par des communautés scientifiques dont les membres participent à la construction et à la circulation de savoirs (Bourdieu, 1976; 1997). Ainsi, les processus de production, diffusion et réception de savoirs structurent l'univers discursif d'espaces académiques, de disciplines et de champs de savoirs. Cette approche de la science comme pratique sociale nous permet d'analyser certains mécanismes propres aux communications scientifiques (comme les citations entre auteurs) qui rendent compte de l'existence de communautés scientifiques «informelles» ou de réseaux virtuels d'acteurs connectés par leurs affinités épistémiques. À partir des travaux de Derek de Solla Price sur les structures des réseaux formés à partir des citations entre articles scientifiques (Price, 1965 ; Price et Beaver, 1966), Diana Crane (1972) a mis en évidence l'existence de « collèges invisibles», qui correspondent à des groupes de collaboration informels qui travaillent sur un thème commun et partagent une même base épistémique. Dans le présent article, nous proposons d'étudier la diffusion et les appropriations du discours du développement durable dans la sphère académique latino-américaine, d'identifier certains noyaux d'acteurs qui forment potentiellement des collèges invisibles (et parfois visibles), et de comprendre dans une certaine mesure les connexions entre les particularités des discours et l'histoire et la réalité socioculturelles de la région.
L'approche par l'analyse de citations a été utilisée pour caractériser le paysage académique de la science de la durabilité à l'échelle mondiale (Kajikawa et al., 2007 ; 2014 ; Bettencourt et Kaur, 2011). D'autres auteurs ont identifié de cette façon les publications les plus influentes dans ce champ de recherche (Schubert et Láng, 2005; Kajikawa et al., 2007 ; 2014 ; Buter et Van Raan, 2012; Quental et Lourenço, 2012; Hassan et al., 2013), voire dans des sous-champs tels que l'économie écologique ou l'économie de l'environnement (Costanza et al., 2004; 2016; Ma et Stern, 2006). Toutefois, si certaines études procédant à une classification des discours environnementaux ont parfois inclus des propositions critiques issues de pays émergents ou en développement (Guha et Martinez-Alier, 1997; Dryzek, 2005; Hopwood et al., 2005), la plupart portent sur les configurations discursives de la pensée occidentale. Cet article retrace les phases de réception et d'appropriation des discours sur la durabilité dans la sphère académique en Amérique latine. Certains auteurs ont analysé la participation latinoaméricaine dans le débat autour de la durabilité soit en proposant une vue d'ensemble (Gudynas, 1999; Heyd, 2005 ; Leff, 2012), soit en se limitant à des discours ou à des auteurs spécifiques (Herrera et al., 2004 ; Eschenhagen, 2012; Rozzi, 2012; Estenssoro, 2015). Cependant, aucune étude quantitative de références alimentant le discours du développement durable en Amérique latine n'a encore été menée.

Suivant l'approche discursive appliquée à la construction de connaissance, la phase de réception du discours du développement durable constitue une phase d'appropriation ou de reconceptualisation. En effet, les acteurs formulent leur réponse au problème de la durabilité en fonction d'évaluations cognitives qui dépendent largement de leur expérience historique et de leur vision du monde. En ce qui concerne les discours académiques, les conceptions du développement durable dépendent entre autres des traditions intellectuelles et politiques dans lesquelles elles s'inscrivent, mais également des interactions et des stratégies des acteurs du champ. En Amérique latine, certains discours académiques du développement durable s'ancrent de ce fait dans l'histoire de la région et reflètent les critiques envers l'ethnocentrisme eurocentrique $^{3}$, ainsi que les luttes pour la reconnaissance et l'autonomie de modèles culturels marginalisés par les

\footnotetext{
${ }^{3}$ L' « eurocentrisme» est un néologisme qui fait référence aux idées qui associent l'histoire et les structures sociales européennes à un modèle universel (considérant les valeurs et l'histoire européennes comme supérieures aux autres). En Amérique latine, plusieurs critiques ont été formulées contre ces idées dont le projet Modernité/colonialité (Enrique Dussel, Anibal Quijano, Walter Mignolo) et la version latinoaméricaine des Subaltern Studies (John Beverley, Robert Carr, Illeana Rodríguez).
} 
colonies et les politiques de développement (entre autres au travers d'institutions internationales tels que la Banque mondiale, le FMI, la Banque interaméricaine pour le développement, etc.). Comme le montre Thomas Heyd (2004; 2005), malgré certaines expériences et propositions communes entre l'Amérique latine et le «Nord global», en Amérique latine, les discours du développement durable sont directement liés à l'expérience de la colonisation, mais aussi à la situation actuelle de la région dans la (semi)périphérie de l'économie monde capitaliste, avec une activité économique fortement centrée sur les ressources primaires et l'extractivisme. Ainsi, des phénomènes comme l'expropriation des terres, l'exploitation par des tiers, l'exposition brutale aux forces du marché mondial sans moyens de contrer les externalités socioéconomiques et environnementales négatives, l'exposition à de multiples pollutions sans restrictions légales contraignantes, l'urbanisation dérégulée, etc. ont généré des cadres culturels particuliers pour l'interprétation de questions socioenvironnementales. Par conséquent, en Amérique latine, certains discours du développement durable reflètent les débats sur le multiculturalisme et la justice sociale et les multiples luttes pour la reconnaissance, en tenant compte des relations de domination instaurées par le capitalisme et la société industrielle (Herrera et al., 1976; Gudynas, 1999; Adams, 2001 ; Rozzi, 2012).

Nous proposons ci-après une cartographie systématique du champ discursif académique du développement durable en Amérique latine à travers différentes périodes. Nous étudierons également les dynamiques des auteurs régionaux dans ces débats ainsi que le rôle vital joué dans certains cas par les institutions qui ont accueilli des projets, des groupes et des programmes de recherche. Ces dernières ont en effet facilité l'émergence d'une «pensée environnementale latino-américaine» (Heyd, 2005; Ferreira et al., 2006; Leff, 2012; Rozzi, 2012), caractérisée par ses tonalités, ses harmonies et ses dissonances par rapport au discours dominant au niveau mondial.

\section{Données et méthodes}

Les dynamiques que nous cherchons à appréhender peuvent être retracées sur la base des productions scientifiques que nous étudierons à partir d'une analyse de réseau (Wasserman et Faust, 1994) fondée sur les outils de la bibliométrie (De Bellis, 2009). Nous utilisons la citation bibliographique pour reconstituer la structure et les caractéristiques du réseau d'acteurs (White, 2011) qui participent au champ discursif académique étudié.

Plus précisément, nous examinons 1) la morphologie et la configuration du réseau; 2) s'il existe une participation active et critique latino-américaine dans le champ discursif du développement durable ; 3) si les auteurs ont développé des interactions endogènes (en Amérique latine même et le cas échéant avec quels pays) ou s'ils ont plutôt privilégié des interactions exogènes (avec des auteurs et des documents de référence en dehors de l'Amérique latine). En outre, nous analysons ce réseau à différents niveaux, sur trois périodes historiques et mettons en évidence certains groupes d'auteurs qui fonctionnent comme "collège invisible».

Les données sur lesquelles se fonde cette analyse proviennent d'un ensemble de documents publiés entre 1970 et 2012 par un échantillon primaire de 93 auteurs latino-américains ${ }^{4}$ (Annexe 1). Ces auteurs ont été sélectionnés à partir d'une revue de la littérature ainsi que par une technique de boule de neige. Pour chaque auteur, un inventaire bibliographique a été réalisé avec le logiciel Publish or Perish (qui utilise la base de données de Google Scholar ${ }^{5}$ ), totalisant 7997 documents ${ }^{6}$. Parmi ces documents, $25,5 \%$ ont pu être utilisés pour extraire manuellement des références bibliographiques, représentant un total de 68459 citations. $16,5 \%$ de ces citations (11242) renvoient directement à des auteurs impliqués dans le champ discursif du développement durable, qu'ils soient latino-américains (7 258 citations) ou étrangers (c'est-à-dire non latino-américains, 3984 citations) $[\text { Tab. 1 }]^{7}$. La liste complète des auteurs et des

\footnotetext{
${ }^{4}$ Notre objectif n'est pas de figer sur leur identité une région du monde ou les auteurs qui pensent depuis ce contexte géographique. Les auteurs latino-américains ne sont pas isolés du reste du monde ni producteurs de sens au sein de limites finies. Toutefois, les auteurs sélectionnés dans l'échantillon identifient l'Amérique latine comme un territoire marqué par une expérience et une tradition intellectuelle qui ont influencé leurs modes d'appropriation du discours du développement durable.

${ }^{5}$ Google Scholar est moins précis et paramétré que WOS ou Scopus, mais son catalogue est plus large et permet de lister des auteurs et des documents qui sont peu publiés dans les revues recensées dans les catalogues WOS ou Scopus. Google Scholar est libre d'accès et répertorie un plus grand volume de littérature dans des langues autres que l'anglais. Il comporte aussi plus de publications dans le domaine des sciences sociales, peu présentes dans WOS et déficitaires également dans Scopus.

${ }^{6}$ La collecte des données a été réalisée dans un délai de trois jours au mois de décembre 2012 afin d'éviter les distorsions entre les auteurs dues aux décalages temporels et aux effets potentiels sur les citations. Pour le traitement des données, nous avons considéré seulement le premier auteur des documents tant pour la construction de la base de données que pour les références citées dans les bibliographies de ces documents.

${ }^{7}$ Notons que ce résultat de $16,5 \%$ inclut les autocitations et que le volume des citations tombe à 9,6\% (6 617 occurrences) quand on élimine les autocitations.
} 
Tab 1. Statistiques descriptives générales du matériel empirique.

\begin{tabular}{l} 
Statistique \\
\hline Échantillon \\
Scientifiques latino-américains \\
Période de publication \\
Documents \\
Liste complète (Google Scholar avec \\
le logiciel Publish or Perish) \\
Documents utilisés pour l'extraction \\
de citations \\
Citations \\
Total \\
\hline Citations dans le champ discursif du développement \\
durable
\end{tabular}

$\begin{array}{lr}\begin{array}{l}\text { Réseau global (réseau latino-américain } \\ \text { + réseau international) }\end{array} & \\ \text { Total } & 11242 \\ \text { Total sans autocitations } & \mathbf{6 6 1 7}^{*} \\ \text { Nombre de connexions } & 2058 \\ \text { Nombre de connexions sans autocitations } & \mathbf{1 9 7 5}^{*} \\ \text { Réseau latino-américain } & \\ \text { Nombre de citations reçues par } & 7258 \\ \text { les auteurs latino-américains } & \\ \text { Nombre de citations reçues par } & \\ \text { les auteurs latino-américains sans } & \\ \text { autocitations } & \\ \text { Nombre de connexions } & \mathbf{2 6 3 3}^{*} \\ \text { Nombre de connexions } & 711 \\ \text { sans autocitations } & \mathbf{6 2 8}^{*} \\ \text { Réseau interntional }\end{array}$

Réseau international

Nombre de citations reçues

par les auteurs internationaux

Nombre de connexions

* Les valeurs indiquées en gras sont celles qui seront le plus utilisées dans les calculs sur les réseaux (soit le total des citations et des connexions de la matrice carrée du réseau latino-américain et de la matrice rectangulaire du réseau global).

documents cités se trouve en annexe 2 ; un identifiant reporté dans les graphiques permet de situer les auteurs dans les différents réseaux.

Afin de structurer le réseau de citations du champ académique du développement durable en Amérique latine, nous avons construit différentes «matrices» (Wasserman et Faust, 1994, p. 150-153) incluant l'échantillon des 93 auteurs latino-américains en tant que «citants» et les auteurs de l'échantillon ainsi que les auteurs étrangers cités par ces derniers en tant que " cités " (N.B. Seuls les auteurs latino-américains sont donc considérés parmi les «citants»). Ainsi nous avons construit un réseau bimodal à partir de deux types de matrices (Fig. 1): une matrice carrée qui inclut exclusivement les auteurs latino-américains (et les citations entre eux; de 1 à $n$ ) et une matrice rectangulaire construite à partir des citations des auteurs latino-américains (de 1 à $n$ ) vers des auteurs et des documents internationaux (c'est-àdire non latino-américains, de 1 à $m$ ). Il s'agit d'un réseau bimodal dans la mesure où il combine les citations des auteurs latino-américains entre eux et les citations des auteurs latino-américains vers des auteurs et des documents étrangers (qui sont considérés comme événements construits à partir d'un lien de citation). Nous n'avons pas considéré les citations des auteurs étrangers (non compris dans l'échantillon primaire). Les liens du réseau sont donc tous de même nature (citant-cité), mais les récepteurs sont différents et peuvent faire partie soit du réseau latinoaméricain, soit du réseau international.

À partir de ces matrices, le logiciel GEPHI ${ }^{8}$ a permis de générer des réseaux, de les visualiser et d'ordonner les auteurs dans un espace euclidien bidimensionnel en fonction d'algorithmes de centralité et de proximité. Nous avons utilisé pour ce faire deux mesures : la mesure de centralité et la mesure d'intermédiation calculées respectivement avec les algorithmes Indegree ${ }^{9}$ et Betweeness ${ }^{10}$ pour discriminer les auteurs les plus importants dans le réseau ${ }^{11}$. Les auteurs «médiateurs » jouent un rôle d'intermédiaires entre les auteurs parce qu'ils permettent de connecter et d'intégrer les auteurs du champ à travers les citations. Les résultats apparaissent sur les figures 2 à 7 ciaprès. Dans ces graphiques, les auteurs sont considérés comme des «nœuds » (les sommets représentés par un point dans les graphiques) et les citations produites par les auteurs latino-américains sont considérées comme des «liens» entre auteurs (les arêtes, représentées par une flèche dans les

\footnotetext{
${ }^{8}$ https://gephi.org/

${ }^{9}$ Le Indegree est le nombre de liens vers le noud calculé à partir de la formule suivante : pour un graphe $\mathrm{G}:=(\mathrm{V}, \mathrm{E})$ avec $n$ sommets, le degré de centralité Indegree $\mathrm{C}_{\mathrm{D}}(\mathrm{v})$ pour un sommet $v$ est égale à $\mathrm{C}_{\mathrm{D}}(v)=\operatorname{deg}(v) / n-1$.

${ }^{10}$ Le Betweenness est une mesure de centralité d'un sommet dans un graphique. Dit simplement, les sommets qui se trouvent sur de nombreux chemins les plus courts entre les autres sommets auront un coefficient Betweenness plus élevé que ceux qui se trouvent sur peu de chemins. Ils jouent en quelque sorte un rôle d'intermédiaire dans le réseau. Le coefficient d'intermédiation (Betweeness) se calcule selon la formule suivante: $\mathrm{C}_{\mathrm{B}}(v)=\sum s \neq v \neq t \epsilon V$.

${ }^{11}$ Dans l'analyse de réseau, la "centralité » renvoie à une mesure sur les nœuds qui détermine leur importance relative dans l'ensemble du réseau (Borgatti, 2005 ; Borgatti et Everett, 2006). Quatre mesures sont généralement utilisées : le degré de centralité («indegree »), de proximité («closeness »), d'intermédiation («betweenness ») et l'eigenvector. Ici nous utilisons le coefficient Indegree et Betweenness pour considérer le degré de centralité et d'intermédiation.
} 


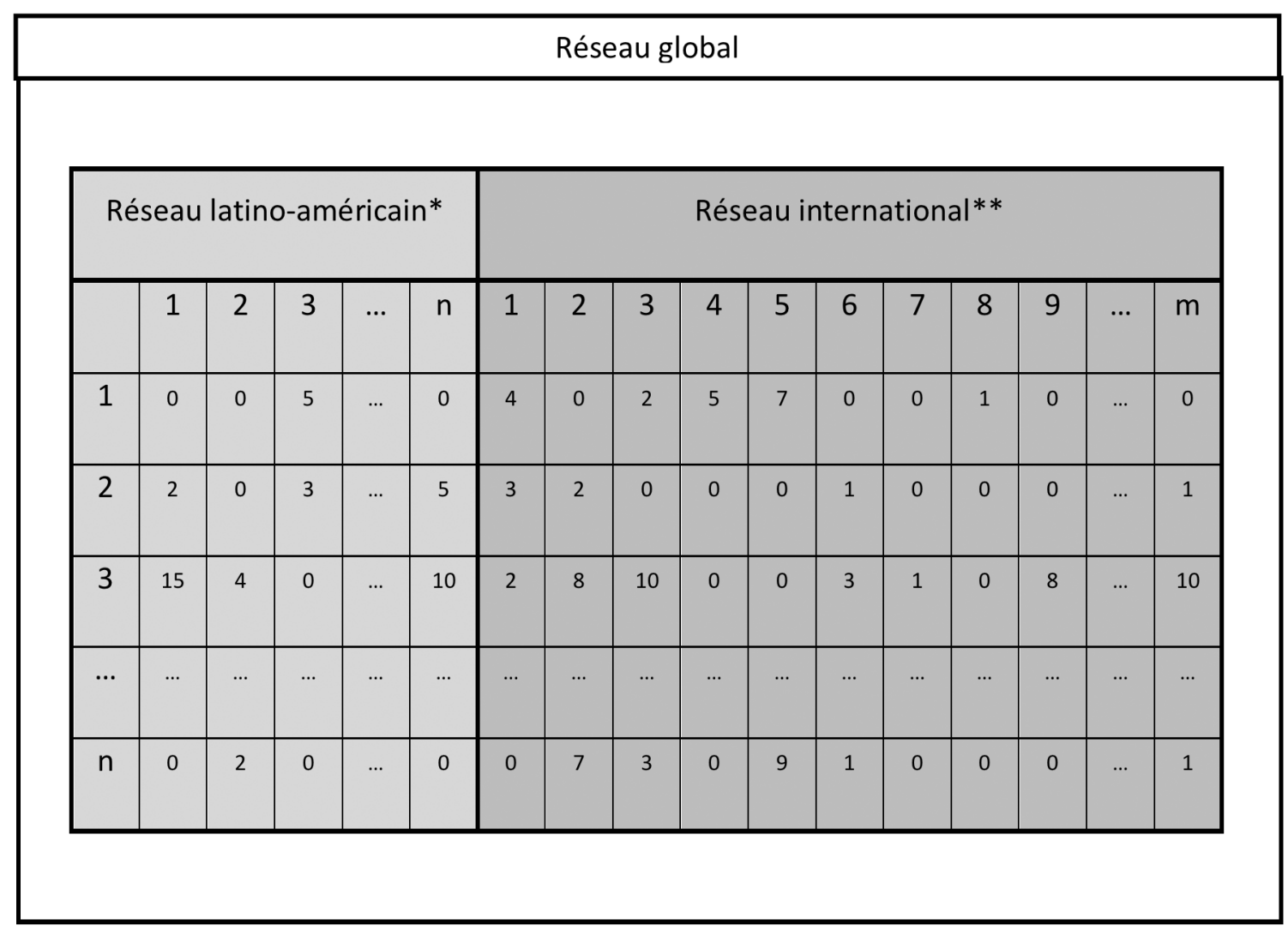

*Citations valuées hypothétiques entre auteurs latino-américains (sans autocitations et potentiellement bidirectionnelles)

**Citations valuées hypothétiques des auteurs latino-américains vers des auteurs et documents internationaux (non latino-américains)

Fig. 1. Schéma (hypothétique) des matrices et des réseaux de citations utilisés pour l'analyse des données (réalisation : J. Vanhulst).

graphiques). Nous pouvons ainsi considérer la participation des auteurs latino-américains dans les débats sur le développement durable ainsi que la centralité relative de ces auteurs dans l'ensemble du réseau de références qu'ils utilisent.

Par ailleurs, à partir de l'analyse de réseau, il est possible de caractériser les acteurs individuels par certains «attributs» qualitatifs qui permettent de distinguer certains «sous-groupes » dans le réseau. Cela permet de caractériser le réseau selon certaines variables qualitatives. Nous avons ainsi défini et codé les auteurs de notre base de données à partir de l'attribut «pays d'origine» et «discipline principale ${ }^{12}$. Cela nous permettra de considérer les pays les plus centraux ainsi que les dialogues interrégionaux, mais aussi de connaître les

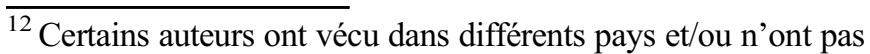
suivi une trajectoire disciplinaire linéaire. Ici, nous avons considéré le pays où ils ont élaboré leur théorie et reçu leur formation initiale. Ainsi, Ignacy Sachs, par exemple, est considéré dans cet article comme brésilien, malgré son appartenance polonaise et française notamment. S'il est difficilement assimilable à un seul pays, il a travaillé et conçu une partie de ses idées sur l'écodéveloppement à partir de son expérience brésilienne.
}

disciplines scientifiques qui participent au champ scientifique du développement durable en Amérique latine et leur évolution historique ${ }^{13}$.

Ces réseaux ont été décomposés en trois périodes : la période pré-Brundtland, la période post-Brundtland et la période contemporaine. La période pré-Brundtland commence, dans notre étude, en 1970 avec l'émergence généralisée des préoccupations environnementales et se termine en 1987 avec la publication du rapport Brundtland (Brundtland et al., 1987). Ce rapport a contribué à la consolidation d'une base institutionnelle pour un développement écologiquement soutenable et à placer la notion de développement durable au centre des débats, influençant fortement le paysage politique et intellectuel (Zaccai, 2002; Schubert et Láng, 2005; Quental et Lourenço, 2012). La période post-Brundtland s'étend de 1988 à 2002 (année du sommet de la Terre à Johannesburg). Certains auteurs ont montré que les pics d'activités académiques dans le domaine du champ discursif du développement

\footnotetext{
${ }^{13}$ Dans la thèse citée en note 1 , on trouvera aussi des analyses semblables avec l'attribut « appartenance disciplinaire», mais nous ne pouvons en reproduire les résultats ici faute de place.
} 


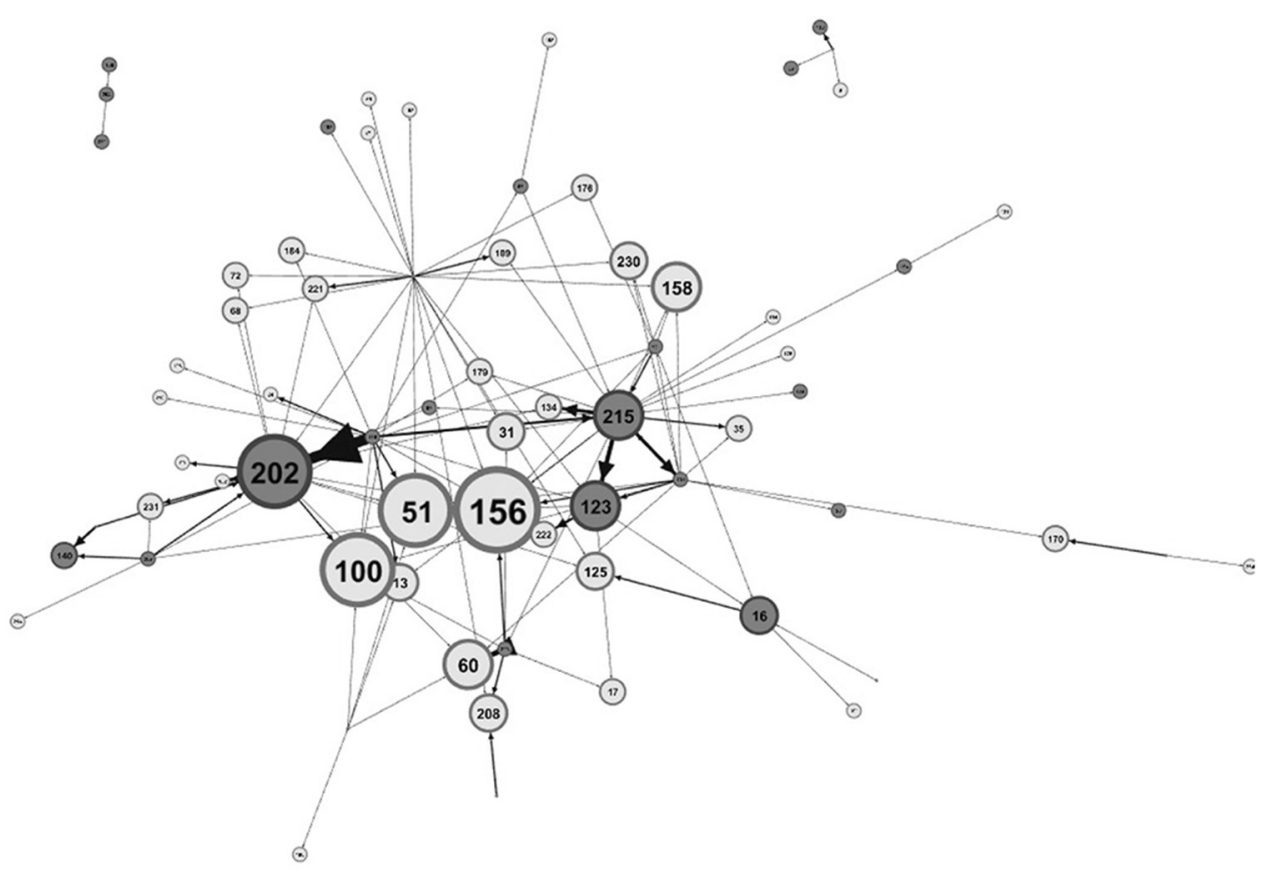

- Auteurs internationaux (non latino-américains)

O Auteurs latino-américains

Fig. 2. Position relative de l'Amérique latine dans le réseau de citations pendant la période 1970-1987 (la taille des nœuds varie selon le coefficient Indegree).

Comme mentionné dans le point sur les données et la méthodologie, les sommets représentent des auteurs, et les flèches représentent une relation entre un auteur citant et un auteur cité (source: d'après Vanhulst et Zaccai, 2016).

durable coïncident avec les sommets internationaux décennaux qui jouent le rôle de catalyseur pour l'action et la réflexion (Hibbard et al., 2007 ; Bettencourt et Kaur, 2011; Quental et al., 2011; Quental et Lourenço, 2012). Enfin, la période contemporaine correspond aux années entre Johannesburg et le sommet de la Terre à Rio de Janeiro en 2012 (Rio+20).

Afin de donner du relief à notre analyse de réseau, nous avons combiné le recours aux méthodes quantitatives avec une analyse de contenu pour une série de discours sélectionnés, limités ici à quelques cas pour des raisons de place. Pour ce faire, nous avons identifié certains auteurs ou groupes d'auteurs centraux ainsi que les documents importants au cours des trois périodes et nous avons combiné une analyse du contenu de ces documents ainsi que de la trajectoire des auteurs.

Nous avons formulé deux hypothèses générales. Premièrement, les intellectuels latino-américains participent de manière active (avec une position centrale dans le réseau régional) et critique (avec des propositions spécifiques en réponse à l'impératif de durabilité) au champ académique du développement durable. Deuxièmement, les différentes définitions du développement durable sont culturellement situées et reflètent, entre autres, les débats dérivés de critiques post-eurocentriques. Nous présentons les résultats de l'analyse dans la partie suivante. Comme nous le verrons, l'approche quantitative (exhaustive sur notre corpus) va nous permettre de tracer la constitution de réseaux d'influences à l'intérieur et à l'extérieur de l'Amérique latine, tandis que l'approche qualitative (sélective) nous montrera la teneur de certains discours choisis parmi les plus centraux dans les réseaux ainsi révélés.

\section{Résultats : les discours du développement durable en Amérique latine}

\section{La période pré-Brundtland (1970-1987)}

Durant cette première période, l'Amérique latine a une position plutôt périphérique. Le réseau est diffus et organisé autour d'un nombre restreint d'auteurs étrangers et latino-américains (en particulier Ignacy Sachs, Amilcar Herrera, Osvaldo Sunkel, David Barkin et Nicolo Gligo) [Tab. 2].

La figure 2 représente le réseau durant la première période. Chaque auteur est représenté par un point (ou « nœud ») dont la taille varie en fonction de son degré de 
Tab 2. Statistiques du réseau correspondant a la période préBrundtland.

\begin{tabular}{llc}
\hline & $\begin{array}{c}\text { Réseau latino- } \\
\text { américain }^{*}\end{array}$ & $\begin{array}{l}\text { Réseau } \\
\text { international }^{* *}\end{array}$ \\
\hline Nombre de nœuds & 25 & 68 \\
Nombre de connexions & 35 & 120 \\
Degré moyen & 0,376 & 0,506 \\
Degré moyen avec poids & 2,56 & 2,603 \\
Diamètre du réseau & 6 & 6 \\
Densité du graphe & 0,058 & - \\
Distance géodésique & 2,649 & 2,572 \\
moyenne & & \\
\hline
\end{tabular}

* Le réseau latino-américain inclut exclusivement les citations entre auteurs latino-américains.

${ }^{* *}$ Le réseau international inclut les citations des auteurs latinoaméricains entre eux et vers des auteurs et des documents internationaux.

${ }^{* * *}$ La densité est calculée uniquement pour le réseau d'auteurs latino-américains (matrice carrée).

centralité (qui permet de distinguer les auteurs les plus centraux). Pour tous les graphiques, les numéros indiqués dans les points correspondent à l'identifiant des auteurs repris en annexe 2. On peut donc se reporter à la liste d'auteurs complète de l'annexe 2 pour situer les auteurs dans le réseau. La figure 3 représente le même réseau et la taille des points varie en fonction du degré d'intermédiation (qui permet de distinguer les auteurs qui jouent un rôle de connecteur dans le réseau, ici limité aux auteurs latino-américains étant donné que nous avons repris exclusivement les références citées par un échantillon d'auteurs latino-américains).

Dans cette première période, les auteurs latinoaméricains les plus centraux sont affiliés à diverses institutions régionales et internationales. Il s'agit par exemple du Bureau régional pour l'Amérique latine et les Caraïbes du Programme des Nations unies pour l'environnement (ORLAC/PNUE), de la Commission économique pour l'Amérique latine et les Caraïbes (CEPAL), de la Fondation Bariloche et de la fondation Dag Hammarskjöld. Ainsi, comme l'a mis en évidence Guillermo Castro Herrera (2000, p. 43) dans son étude sur l'histoire environnementale en Amérique latine: «Avant les années 1970 en Amérique latine, l'intérêt public pour les problèmes environnementaux se résume à quelques préoccupations dispersées et mesures isolées. À partir de cette date, l'incorporation de la thématique "depuis l'extérieur" et "d'en haut" à la vie publique de la région est le résultat de l'intérêt, d'abord d'organismes internationaux du système des Nations unies, et ensuite, du système financier international ».

En 1970, suite à une réunion qui s'est tenue à Rio de Janeiro pour présenter et discuter le World 3 model conçu par une équipe du MIT (et utilisé dans le rapport Halte à la croissance? de Meadows et al., 1972), certains représentants de la délégation latino-américaine adoptent une posture critique et décident de mandater la fondation Bariloche pour construire un modèle alternatif en réponse au modèle du MIT qu'ils considèrent comme incompatible avec la réalité de la "périphérie» latinoaméricaine. Entre 1972 et 1975, un groupe de scientifiques mené par Amilcar Herrera travaille, sous l'égide institutionnelle de la fondation Bariloche, à l'élaboration de ce qui sera appelé plus tard le «modèle mondial latino-américain» ou «modèle Bariloche». Le rapport de la fondation Bariloche est finalement publié en 1976 (Herrera et al., 1976). De façon très succincte, la construction du modèle Bariloche part du principe général que les principaux obstacles au développement harmonieux de l'humanité ne sont pas tant physiques que d'ordre social et politique et qu'ils dépendent d'abord de la répartition du pouvoir à la fois au niveau international, régional et national. Il s'inscrit donc directement dans une discussion sur les asymétries de pouvoir mises en évidence notamment dans la théorie de la dépendance.

Les premiers auteurs centraux sont plutôt éloignés les uns des autres dans le réseau ${ }^{14}$ (à l'exception de Nicolo Gligo et Osvaldo Sunkel qui ont forgé une relation réciproque ${ }^{15}$ encore peu consistante à l'époque, mais qui préfigure une collaboration majeure qui naît en 1978 avec l'unité Développement et environnement au sein de la CEPAL et du ORLAC/PNUE). Ils sont toutefois connectés ${ }^{16}$ non seulement à un certain nombre d'auteurs périphériques mais également à des rapports internationaux emblématiques (comme les deux rapports du Club de Rome : Meadows et al., 1972; Mesarović et Pestel, 1974).

Parmi ces auteurs centraux, on retrouve au premier rang Ignacy Sachs, économiste d'origine polonaise, naturalisé français et exilé au Brésil entre 1941 et 1953. Dans le sillon tracé par le rapport Founex (Strong et al., 1971) et la conférence de Stockholm (1972) ${ }^{17}$, l'approche heuristique

\footnotetext{
${ }^{14}$ En effet, le réseau, avec $75 \%$ de relations infinies, seulement $12,5 \%$ de connexions possibles sans intermédiaires ou avec un intermédiaire ainsi qu'une distance géodésique moyenne de 2,5 , est très peu connecté.

${ }^{15}$ Nous considérons qu'une relation est réciproque quand deux auteurs se citent mutuellement. Dans le cas contraire, la relation est dite unilatérale.

${ }^{16} 36 \%$ des citations émises se réfèrent aux auteurs latinoaméricains, principalement Ignacy Sachs, Amilcar Herrera (presque exclusivement pour le rapport Modèle mondial latino-américain), Osvaldo Sunkel et David Barkin. Les autres auteurs relevés dans la figure 1 jouent un rôle central en tant qu'intermédiaires (émetteurs de citations).

${ }^{17}$ On pourrait aussi ajouter la déclaration de Cocoyoc (1974) et le rapport de la fondation Dag Hammarskjöld What now? (1975) pour lequel Ignacy Sachs était conseiller principal du directeur Marc Nerfin.
} 


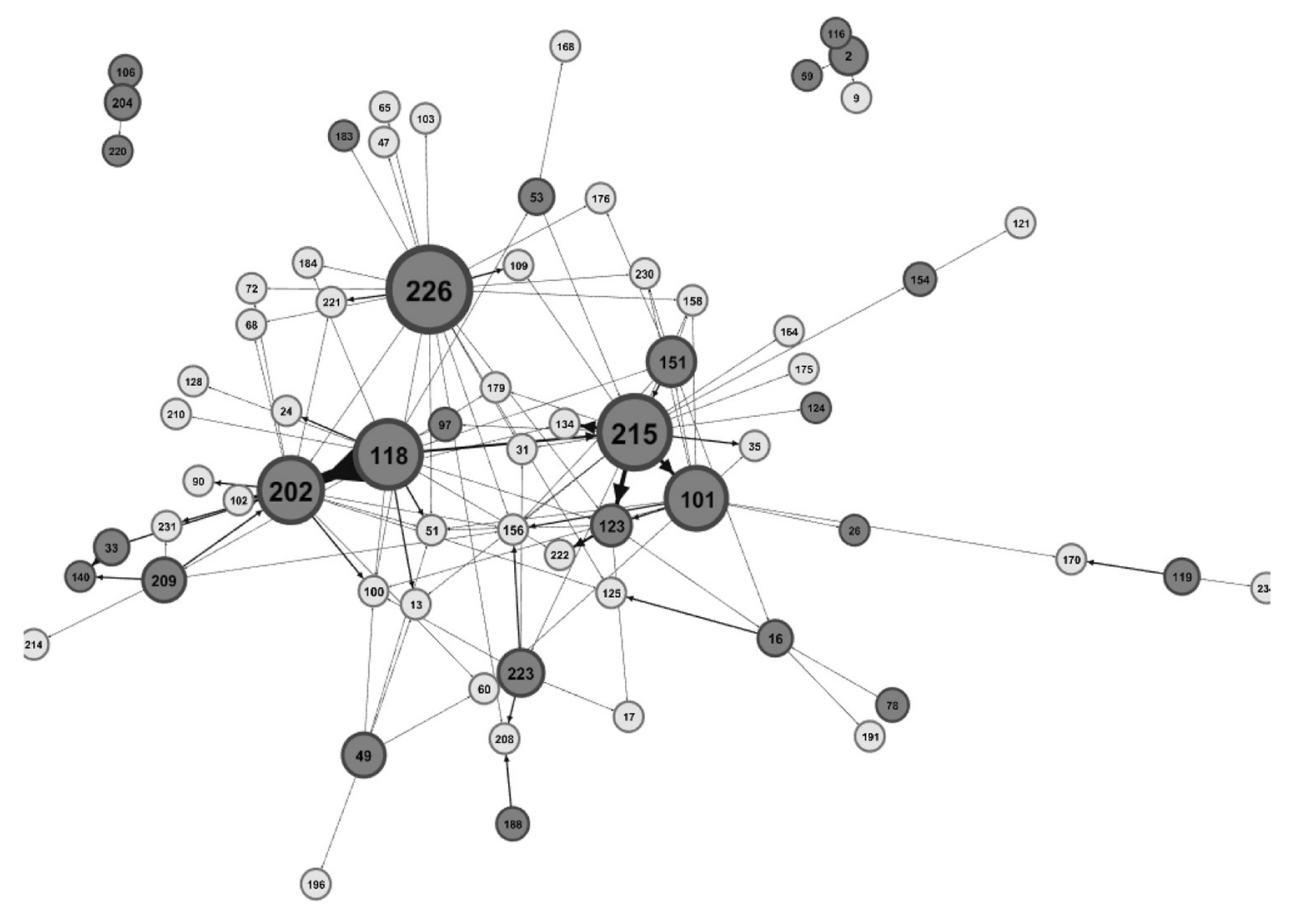

- Auteurs internationaux (non latino-américains)

Auteurs latino-américains

Fig. 3. Auteurs latino-américains intermédiaires dans le réseau de citations pendant la période 1970-1987 (la taille des nœuds varie selon le coefficient Betweenness) (source : d'après Vanhulst et Zaccai, 2016).

de l'écodéveloppement proposée par Sachs a eu un grand impact en Amérique latine. Non seulement à travers de multiples conférences et quelques publications centrales (Sachs I., 1974b ; 1974a), mais aussi grâce à différentes participations d'intellectuels latino-américains au séminaire d'écodéveloppement donné par Ignacy Sachs au Centre international de recherche sur l'environnement et le développement (Cired), fondé à Paris en 1973 par Sachs lui-même. Plus fondamentalement, l'écodéveloppement était perçu en Amérique latine comme une occasion de concilier développement économique et politique environnementale en considérant la situation de la périphérie de la région dans le système économique mondial (Prebisch, 1950). Plutôt qu'un appel à stopper la croissance, c'est un appel à un autre développement qui n'efface pas du débat les structures asymétriques des relations de pouvoir et met au centre la crise environnementale comme potentiel pour réorienter le développement. Ignacy Sachs considérait d'ailleurs la région comme une terre fertile pour accueillir ses propositions. Durant les années 1970, il était présent dans différents pays latinoaméricains pour promouvoir l'écodéveloppement ${ }^{18}$.

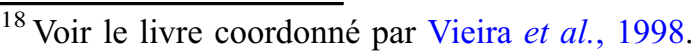

À partir de la morphologie du réseau on peut remarquer que dans cette première période, les auteurs latino-américains citent plutôt des auteurs non latinoaméricains, même si les relations privilégiées sont plutôt internes. Grâce à cette analyse de citations on constate aussi, durant cette période où le champ discursif régional et ses noyaux épistémiques sont encore largement en construction, que les auteurs étrangers sont influents. Par ailleurs, le rôle d'intermédiaire joué par certaines des plus importantes institutions publiques latino-américaines dans le cadre de la promotion du débat autour de la durabilité mérite d'être souligné.

À partir des analyses en fonction des attributs (pays d'origine des auteurs et discipline principale), nous pouvons également apprécier certaines caractéristiques particulières de l'évolution du champ des débats académiques du développement durable en Amérique latine. Les pays latino-américains qui participent le plus activement à ces discussions sont l'Argentine, le Brésil, le Chili et le Mexique. Ces pays centraux ont été les lieux historiques où se sont développés des centres de recherche et d'éducation autour des questions du développement et de l'environnement, s'affirmant ainsi comme centres névralgiques de réflexion à l'échelle régionale. Les participants au réseau dans cette première période sont 


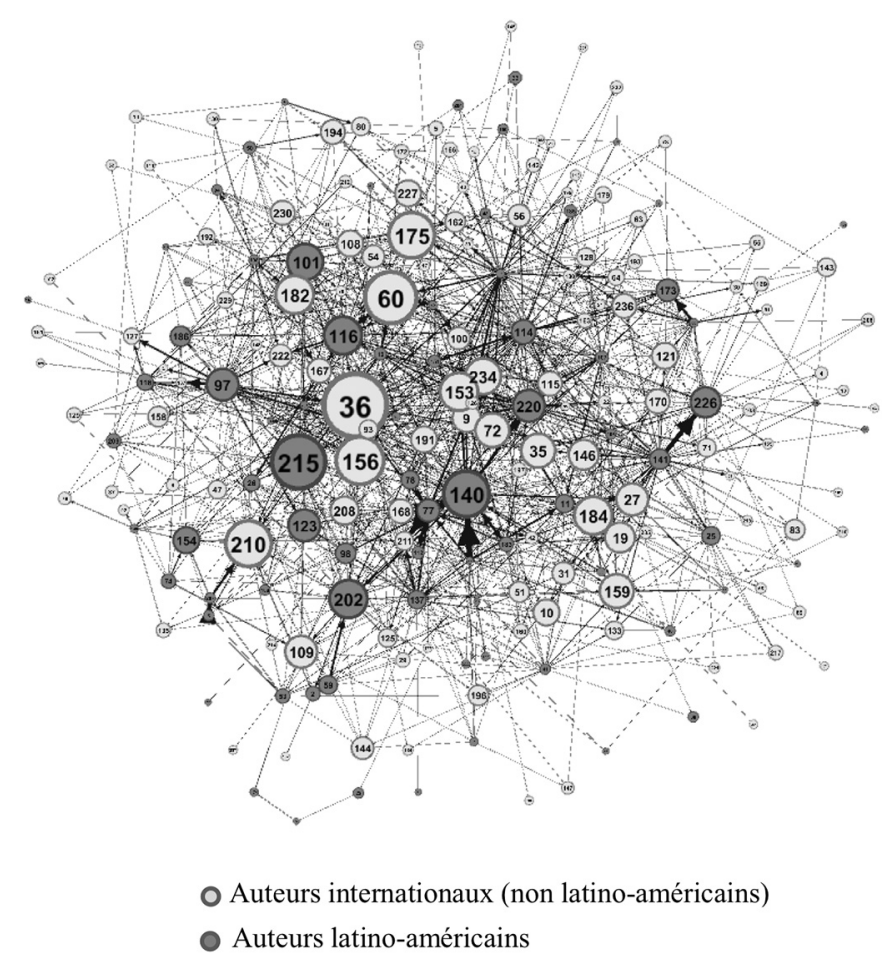

Fig. 4. Position relative de l'Amérique latine dans le réseau de citations pendant la période 1988-2002 (la taille des nœuds varie selon le coefficient Indegree) (source : d'après Vanhulst et Zaccai, 2016).

essentiellement économistes ou spécialistes des sciences naturelles, ce qui contribue à expliquer la prépondérance de l'économie (critique), des sciences naturelles et des rapports internationaux dans les références internationales. Les autres sciences humaines et sociales sont quant à elles pratiquement absentes du débat.

\section{La période post-Brundtland (1988-2002)}

Durant la seconde période, «post-Brundtland», le champ discursif du développement durable en Amérique latine se consolide rapidement (Tab. 3).

Comme nous pouvons l'observer sur la figure 4 qui représente le réseau durant la seconde période, le réseau s'intensifie et prend une forme concentrique. Il ne s'agit plus désormais de quelques centres disparates autour desquels s'organise le réseau, mais d'un ensemble plus homogène, composé d'auteurs centraux et périphériques. Sur la figure 5 qui représente le même réseau, la taille des points varie en fonction du degré d'intermédiation (lequel permet de distinguer les auteurs qui jouent un rôle de connecteur dans le réseau, ici limité aux auteurs latino-américains étant donné que nous avons repris exclusivement les références citées par un échantillon d'auteurs latino-américains).

Les auteurs latino-américains forgent petit à petit un champ discursif régional plus intégré. Ainsi, même si dans l'absolu le volume de citations est plus important
Tab 3. Statistiques du réseau correspondant a la période postBrundtland.

\begin{tabular}{lcc}
\hline & $\begin{array}{c}\text { Réseau latino- } \\
\text { américain }^{*}\end{array}$ & $\begin{array}{l}\text { Réseau } \\
\text { international }^{* *}\end{array}$ \\
\hline Nombre de nœuds & 75 & 224 \\
Nombre de connexions & 317 & 1068 \\
Degré moyen & 4,227 & 4,768 \\
Degré moyen avec poids & 12,173 & 11,902 \\
Diamètre du réseau & 7 & 8 \\
Densité du graphe & 0,057 & - \\
Distance géodésique & 2,91 & 2,857 \\
moyenne & & \\
\hline
\end{tabular}

${ }^{*}$ Le réseau latino-américain inclut exclusivement les citations entre auteurs latino-américains.

${ }^{* *}$ Le réseau international inclut les citations des auteurs latinoaméricains entre eux et vers des auteurs et des documents internationaux.

${ }^{* * *}$ La densité est calculée uniquement pour le réseau d'auteurs latino-américains (matrice carrée).

vers les auteurs internationaux, seuls deux auteurs latinoaméricains (Hector Sejenovich et José Sarukhan) parmi les 68 auteurs actifs au cours de cette seconde période dialoguent exclusivement avec des auteurs internationaux et non latino-américains. Tous les autres citent à la fois des auteurs internationaux et des auteurs latinoaméricains. 


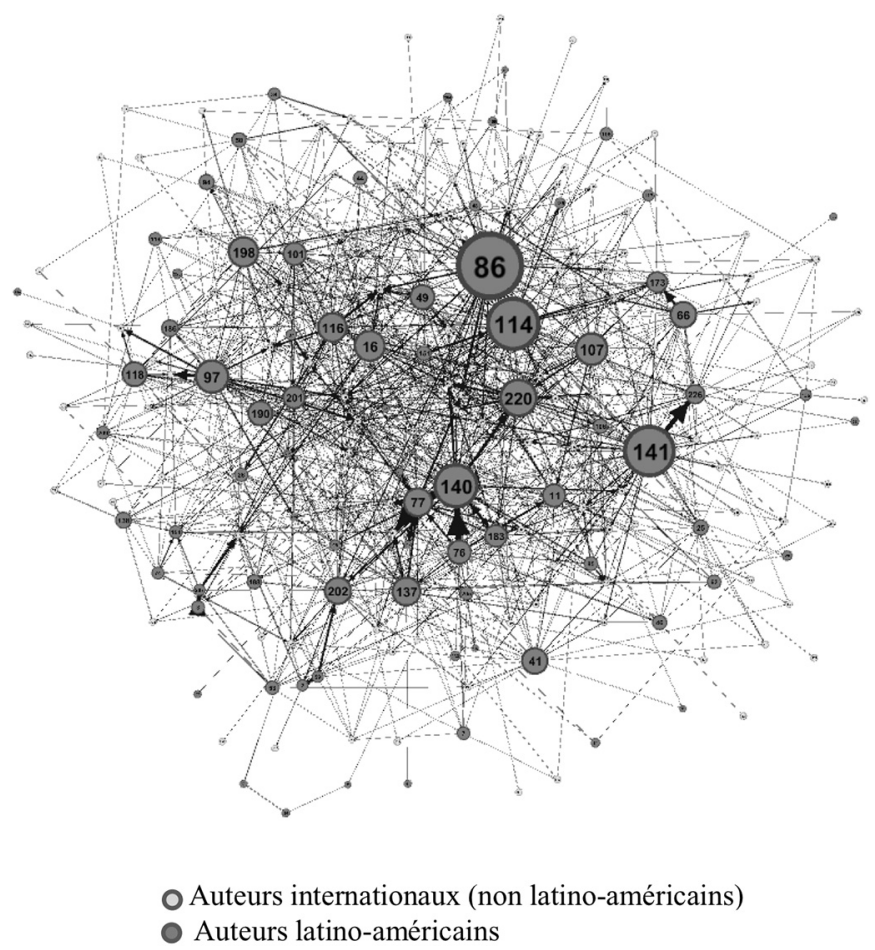

Fig. 5. Auteurs latino-américains intermédiaires dans le réseau de citations pendant la période 1988-2002 (la taille des nœuds varie selon le coefficient Betweenness) (source : d'après Vanhulst et Zaccai, 2016).

La relation réciproque la plus importante naît de la consolidation du lien entre Enrique Leff et Arturo Escobar (qui deviendront les auteurs les plus influents au cours de la troisième période). Cette relation est moins institutionnelle qu'épistémique, liée au type de discours radical et anti-hégémonique que partagent les deux auteurs. Selon le Mexicain Enrique Leff (2004), « la rationalité environnementale» doit aller au-delà de la mise à disposition d'un ensemble d'outils pour la gestion efficace de l'environnement: elle s'oppose diamétralement à la «rationalité économique» dominante en Occident et cherche à guider la praxis à partir de la subversion des principes qui régissent et légitiment la rationalité théorique et instrumentale de la modernité ${ }^{19}$. Leff appelle à une nouvelle rationalité qui intégrerait les valeurs, la raison et le sens, qui s'ouvrirait à la différence et à la diversité, dans le but de déconstruire la logique totalisante de la modernité eurocentrique. Les synergies avec la proposition du «post-développement» du Colombien Artur Escobar $(1992 ; 1994)$ formé et enseignant en partie aux États-Unis sont évidentes.

Nous pouvons également souligner l'importance de la relation réciproque entre Enrique Leff et Carlos Walter Porto Gonçalves, un géographe brésilien qui s'inscrit dès ses premiers travaux dans le mouvement de géographie

\footnotetext{
${ }^{19}$ Notons qu'Enrique Leff associe de façon synonymique la «rationalité économique» à la modernité, ici réduite à sa version universaliste et eurocentrique.
}

critique. Gonçalves a développé l'idée d'une «géographie environnementale» (Gonçalves, 2001) à partir de ses travaux avec les seringueiros (récolteurs de caoutchouc) de la forêt amazonienne, et notamment de sa collaboration avec son ami et collègue Francisco «Chico» Mendes à Xapuri. Cette perspective s'apparente beaucoup à celles d'Enrique Leff et d'Arturo Escobar, et ensemble, ils articulent un collège invisible qui prendra de plus en plus d'ampleur au cours de la période contemporaine, et qui s'inscrit dans une perspective de "transformation » critique des propositions réformistes et du statu quo.

Au cours de cette période, les auteurs latino-américains colonisent le centre et deviennent plus présents. Neuf auteurs latino-américains partagent le centre avec les auteurs internationaux. Les relations entre les auteurs latino-américains et les auteurs étrangers se fortifient également. Les trois auteurs latino-américains qui étaient centraux dans la première période (Ignacy Sachs, Amílcar Herrera et Osvaldo Sunkel) renforcent leur position. Le rapport Brundtland est devenu une référence incontournable immédiatement après sa publication, notamment pour les auteurs principaux (Gilberto Gallopin, Victor Toledo, Eduardo Viola et Enrique Leff), mais aussi pour Manfred Max-Neef (dans ses propositions portant sur un développement à échelle humaine) ainsi que pour Roberto Guimarães et Nicolo Gligo, qui tous deux sont liés à la CEPAL et expriment une critique virulente du modèle néolibéral (Gligo, 2001 ; Guimarães, 2003). 


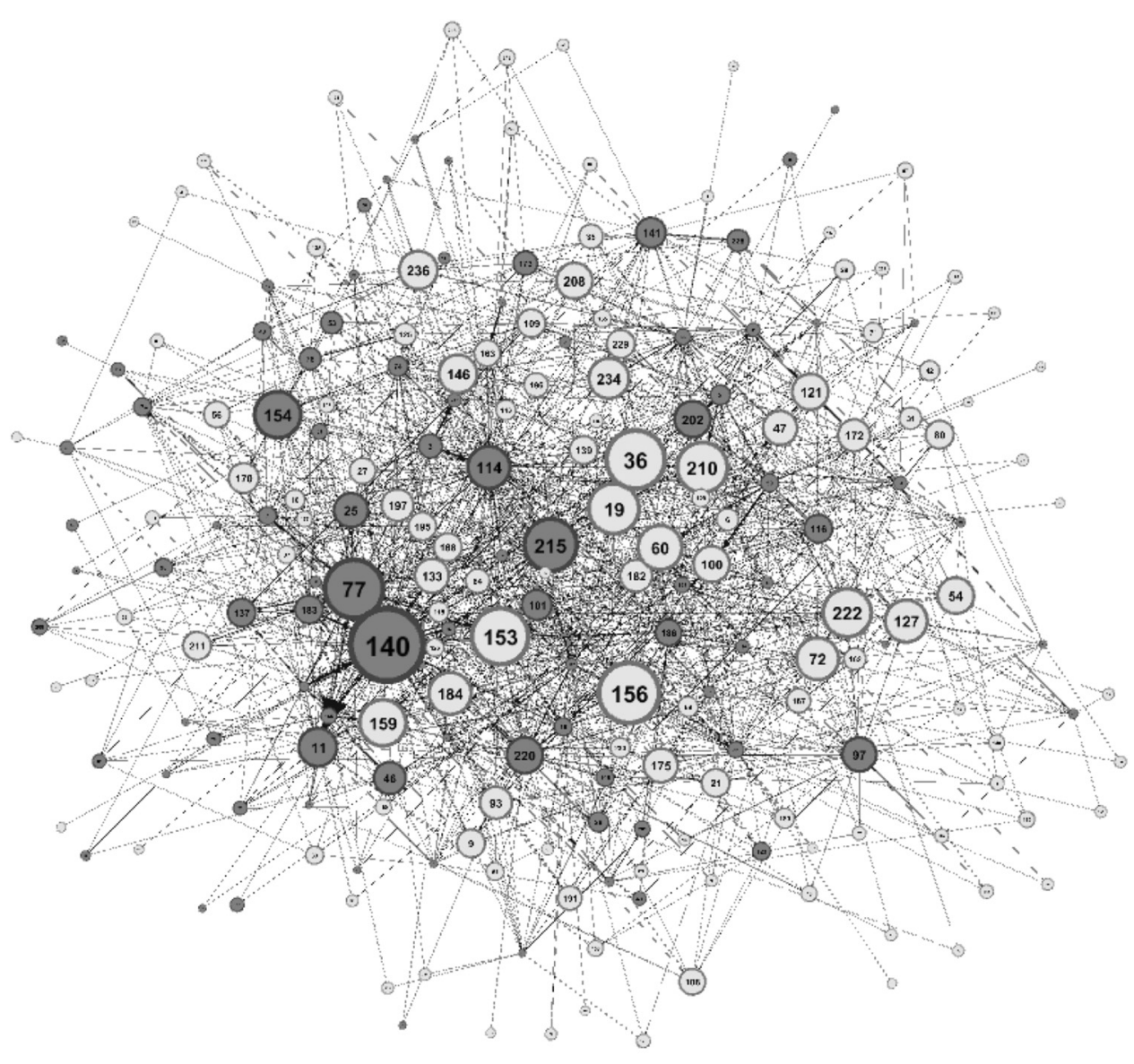

O Auteurs internationaux (non latino-américains)

Auteurs latino-américains

Fig. 6. Position relative de l'Amérique latine dans le réseau de citations pendant la période 2003-2012 (la taille des nœuds varie selon le coefficient Indegree) (source: d'après Vanhulst et Zaccai, 2016).

\section{La période « contemporaine » (2003-2012)}

Globalement, cette troisième période suit les tendances tracées durant la seconde période : 1) l'intensification du champ; 2) la cohérence relative et la forme concentrique du champ; 3) la centralité des auteurs d'Amérique latine; 4) les relations privilégiées entre auteurs latino-américains; 5) la centralité des auteurs issus du Brésil, du Mexique, de la Colombie, du Chili et de 1'Argentine (Tab. 4 ; fig. 6 ; fig. 7).

Le champ discursif du développement durable se consolide en Amérique latine et la région partage le centre du réseau avec les rapports et les auteurs internationaux : en valeur relative (nombre de connexions), 60\% des connexions se dirigent vers l'extérieur de l'espace latino-américain contre $40 \%$ pour le réseau interne. Ces chiffres témoignent d'une participation active dans l'élaboration du discours du développement durable en Amérique latine (au moins, au niveau académique).
Tab 4. Statistiques du réseau correspondant a la période contemporaine.

\begin{tabular}{lcc}
\hline & $\begin{array}{c}\text { Réseau latino- Réseau } \\
\text { américain }^{*}\end{array}$ & $\begin{array}{l}\text { international } \\
\text { i** }\end{array}$ \\
\hline Nombre de nœuds & 87 & 216 \\
Nombre de connexions & 447 & 1302 \\
Degré moyen & 5,138 & 6,028 \\
Degré moyen avec poids & 19,034 & 17,481 \\
Diamètre du réseau & 7 & 7 \\
Densité du graphe & 0,06 & - \\
Distance géodésique moyenne & 2,717 & 2,726 \\
\hline
\end{tabular}

${ }^{*}$ Le réseau latino-américain inclut exclusivement les citations entre auteurs latino-américains.

${ }^{* *}$ Le réseau international inclut les citations des auteurs latinoaméricains entre eux et vers des auteurs et des des documents internationaux. 


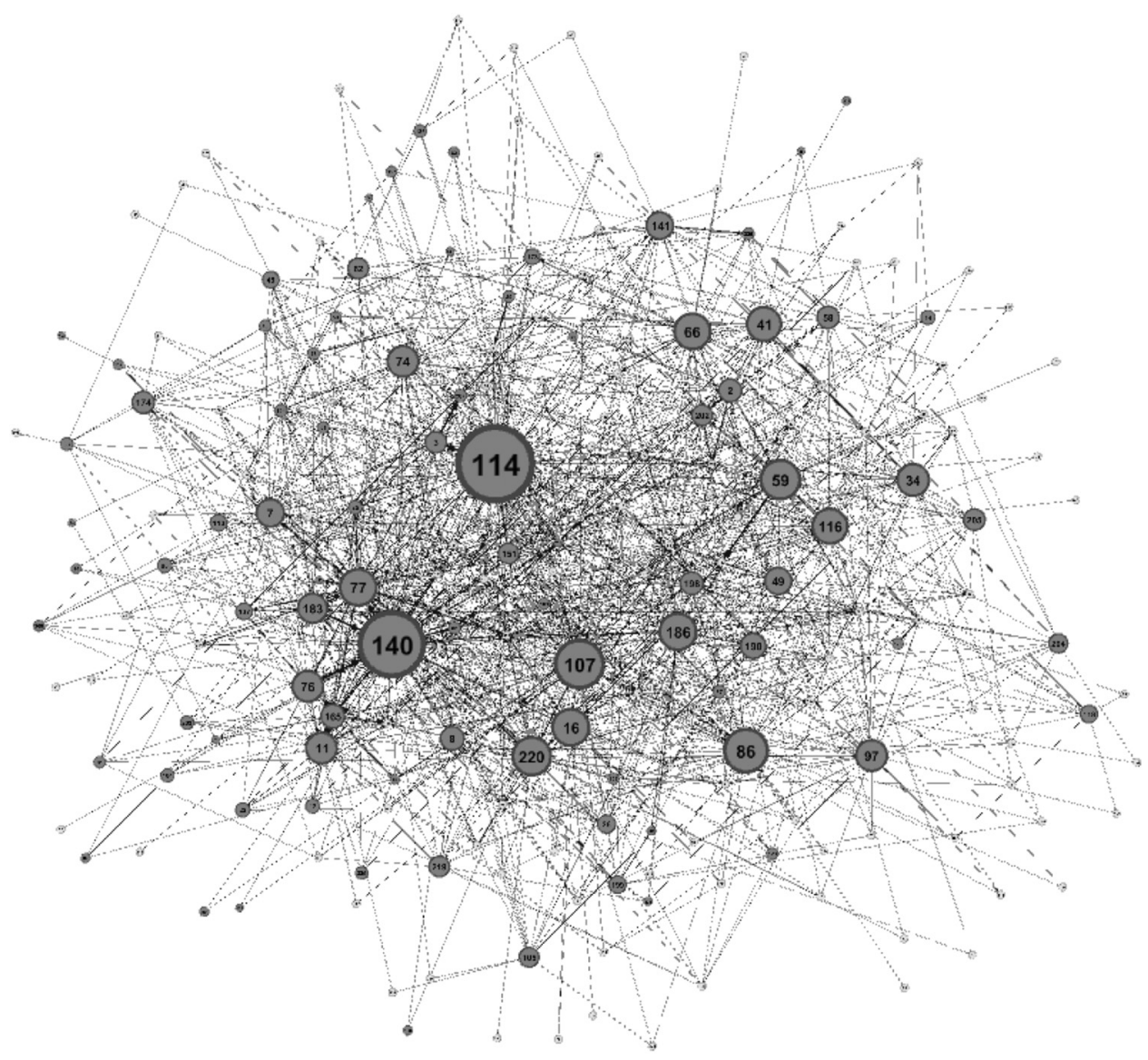

O Auteurs internationaux (non latino-américains)

- Auteurs latino-américains

Fig. 7. Auteurs latino-américains intermédiaires dans le réseau de citations pendant la période 1988-2002 (la taille des nœuds varie selon le coefficient Betweenness) (source : d'après Vanhulst et Zaccai, 2016).

La Colombie se démarque pendant cette troisième période, à la faveur de l'activité soutenue de philosophes colombiens et de la création de l'Instituto de Estudios Ambientales (IDEA, Institut des études de l'environnement). En effet, à partir de la fin des années 1980, plusieurs instituts sont créés pour répondre au défi de la durabilité ; c'est le cas de l'IDEA au sein de l'Université nationale en 1989 à Bogotá, puis en 1991 à Manizales sous la direction d'Augusto Angel Maya. Ils adoptent un discours critique face au réductionnisme et au technocentrisme attribué au discours eurocentrique. On retrouve cette pensée et les discours philosophico-éthiques du groupe IDEA dans les travaux d'Augusto Angel Maya et de Julio Carrizosa, mais aussi chez Ana Patricia Noguera et María Luisa Eschenhagen, héritières de ces précurseurs colombiens.

$\mathrm{Au}$ cours de cette troisième période, Enrique Leff renforce sa position centrale dans le réseau, au-dessus de tout auteur étranger ou de tout rapport international. Cet intellectuel a joué un rôle fondamental dans le développement et la consolidation d'un réseau de formation environnementale en Amérique latine, dans l'inclusion de l'interdisciplinarité dans la recherche en environnement et dans la stimulation de la pensée environnementale latino-américaine, principalement depuis le Programme des Nations unies pour l'environnement (PNUE) et l'Universidad National Autónoma de México (Leff, $1986 ; 1994 ; 2004 ; 2015)$. Les rapports Brundtland et Meadows restent néanmoins des références centrales, à côté des rapports du GIEC (Groupe d'experts intergouvernemental sur l'évolution du climat). À l'image de la seconde période, les relations privilégiées se développent beaucoup entre des auteurs latino-américains. En effet, les relations les plus fortes se situent au niveau régional, comme celles entre Ana Patricia Noguera et Augusto Angel Maya (à l'IDEA) ou entre Alberto Acosta et Eduardo Gudynas autour du discours du Buen Vivir, littéralement le Bien Vivre. 
Le Buen Vivir constitue une traduction espagnole controversée de la notion de sumak kawsay, en quechua (et de notions similaires d'autres peuples autochtones d'Amérique latine), qui signifie en termes généraux « vivre en harmonie et en équilibre, en harmonie avec les cycles de la Terre-Mère, du cosmos, de la vie et de l'histoire, et en équilibre avec toute forme d'existence» (Huanacuni Mamani, 2010, p. 7). Le discours du Buen Vivir, installé dans le débat public régional dans les années 2000 (Vanhulst, 2015), est souvent défini comme alternative au développement, remettant en cause non seulement ce discours mais aussi, plus largement, la matrice socioculturelle de la modernité eurocentrique (Vanhulst et Beling, 2013a). De fait, le Buen Vivir synthétise la proposition collective d'un nouveau modèle culturel: une vision de la bonne société qui revoit les cadres qui caractérisent les institutions et les valeurs politiques et culturelles modernes (capitalisme, industrialisme, universalisme, dualisme, cartésianisme, etc.), et simultanément (ré)introduit et souligne les notions de valeur intrinsèque de la nature et son lien symbiotique avec les sociétés humaines, le collectif comme condition de possibilité pour l'individu, et les dimensions immatérielles du bien-être humain, entre autres ${ }^{20}$.

\section{Conclusion}

Dans cet article, nous avons procédé à une analyse approfondie des phases de réception et d'appropriation/ reformulation des discours académiques sur le développement durable en Amérique latine sur plus de quatre décennies.

Nos résultats quantitatifs montrent que la région latinoaméricaine a participé aux débats sur les liens entre environnement et développement au moins depuis les années 1970 et que le réseau académique s'est consolidé au fil du temps. Le nombre d'acteurs qui y participent a progressivement augmenté, créant des connexions de plus en plus robustes. Les auteurs centraux et les médiateurs ainsi que les institutions ayant accueilli des projets, des groupes de recherche et différents types de programmes ont ainsi joué un rôle central dans la construction d'une «pensée environnementale latino-américaine ». Les résultats indiquent également une polarité internationale forte. En dehors de l'Amérique latine, la quasi-totalité des références sont dirigées vers des auteurs issus des ÉtatsUnis et d'Europe, reproduisant les structures mondiales de la production et de la circulation scientifiques.

L'analyse qualitative, via le contenu des travaux des auteurs latino-américains les plus cités, établit que les

\footnotetext{
${ }^{20}$ Pour plus de précisions sur ce discours nous renvoyons aux travaux d'Eduardo Gudynas et Alberto Acosta (Gudynas, 2011; Gudynas et Acosta, 2011) et à Vanhulst et Beling (2013b, 2014; 2017).
}

auteurs centraux en Amérique latine adoptent souvent une position critique et ce en accord avec différents discours internationaux (ceux de l'économie écologique, de l'écologie politique ou de la décroissance, pour ne citer qu'eux) qui prônent une transformation sociétale. D'autres discours influents, surtout durant la première période, adoptent des visions plus classiques.

Dès lors, même si les stratégies mises en place au niveau des politiques publiques et des entreprises s'accordent plutôt avec le discours dominant, plus proche du statu quo ou de la réforme progressive, les discours critiques, souvent construits en synergie avec certains mouvements sociaux et parfois même adoptés par les politiques publiques, comme c'est partiellement le cas pour le Buen Vivir, sont bien présents dans ce champ académique. Nous pouvons donc confirmer l'hypothèse d'une participation active et critique de l'Amérique latine au champ discursif du développement durable.

Comme nous l'avons signalé en introduction, dans ce sous-continent, les discours du développement durable sont singulièrement mêlés à l'expérience de la colonisation et à ses conséquences sociales, économiques, environnementales et culturelles. Il ressort de plusieurs analyses de contenu que les discours du développement durable en Amérique latine reflètent les débats du multiculturalisme et les multiples luttes pour la reconnaissance, la justice sociale et la validité de modèles culturels marginalisés par la modernité eurocentrique. Notre étude confirme que les significations du développement durable sont culturellement situées (contrairement à un postulat universaliste en matière de discours) et reflètent, entre autres, les débats issus des critiques post-eurocentriques et post-structuralistes.

L'objectif principal de cet article était d'analyser, de structurer et de cartographier la participation latinoaméricaine dans le champ discursif du développement durable. Des recherches plus poussées sur les institutions, auteurs centraux et médiateurs ainsi qu'une analyse plus étendue des liens entre discours et contextes socioculturels pourraient être menées. De plus, l'introduction de variables qualitatives reflétant les disciplines des auteurs pourrait conduire à des résultats intéressants. Dans une perspective plus contextuelle, une analyse approfondie des liens entre les manières de problématiser la modernité dans ces discours et la spécificité du contexte latino-américain, s'ajoutant aux dimensions sociales, économiques et environnementales, pourrait enrichir les réflexions portant sur la dimension culturelle du développement durable.

\section{Références}

Adams W.M., 2001 [ $^{\text {st }}$ ed. 1990]. Green development: environment and sustainability in the Third World, London/ New York, Routledge. 
Bettencourt L.M.A., Kaur J., 2011. Evolution and structure of sustainability science, Proceedings of the National Academy of Sciences, 108, 49, 19540-19545, doi: 10.1073/ pnas.1102712108.

Borgatti S.P., 2005. Centrality and network flow, Social Networks, 27, 1, 55-71, doi: 10.1016/j.socnet.2004.11.008.

Borgatti S.P., Everett M.G., 2006. A Graph-theoretic perspective on centrality, Social Networks, 28, 4, 466484, doi: 10.1016/j.socnet.2005.11.005.

Bourdieu P., 1976. Le champ scientifique, Actes de la recherche en sciences sociales, 2, 2-3, 88-104, doi: 10.3406/arss.1976.3454.

Bourdieu P., 1997. Les usages sociaux de la science: pour une sociologie clinique du champ scientifique, Versailles, Quæ.

Brundtland G.H., Gagon L., Mead H.L. (Eds), 1987. Notre avenir à tous. Rapport de la Commission mondiale sur l'environnement et le développement de l'Organisation des Nations unies, Montréal, Éditions du Fleuve.

Buter R.K., Van Raan A.F.J., 2012. Identification and analysis of the highly cited knowledge base of sustainability science, Sustainability Science, 8, 2, 253-267, doi: 10.1007/ s11625-012-0185-1.

Castro Herrera G., 2000. La crisis ambiental y las tareas de la historia en América Latina, Papeles de Población, 6, 24, 37-60.

Connelly S., 2007. Mapping sustainable development as a contested concept, Local Environment, 12, 3, 259-278, doi: 10.1080/13549830601183289.

Costanza R., Stern D., Fisher B., He L., Ma C., 2004. Influential publications in ecological economics: a citation analysis, Ecological Economics, 50, 3-4, 261-292, doi: 10.1016/j.ecolecon.2004.06.001.

Costanza R., Howarth R.B., Kubiszewski I., Liu S., Ma C., Plumecocq G., Stern D.I., 2016. Influential publications in ecological economics revisited, Ecological Economics, 123, 68-76, doi: 10.1016/j.ecolecon.2016.01.007.

Crane D., 1972. Invisible colleges. Diffusion of knowledge in scientific communities, Chicago, University of Chicago Press.

De Bellis N., 2009. Bibliometrics and citation analysis: from the science citation index to cybermetrics, Lanham/ Toronto/Plymouth, Scarecrow Press.

Dryzek J.S., 2005 [ $^{\text {st }}$ ed. 1997]. The politics of the earth. Environmental discourses, Oxford, Oxford University Press.

Eschenhagen M.L., 2012. Aproximaciones al pensamiento ambiental de Enrique Leff, Environmental Ethics, 34, Supplement, 89-95, doi: 10.5840/enviroethics201234Sup plement57.

Escobar A., 1992. Imagining a post-development era? Critical thought, development and social movements, Social Text, 31/32, 20-56, doi: 10.2307/466217.

Escobar A., 1994. Encountering development. The making and unmaking of the Third World, Princeton, Princeton University Press.

Estenssoro F., 2015. El ecodesarrollo como concepto precursor del desarrollo sustentable y su influencia en América
Latina, Universum, 30, 1, 81-99, doi: 10.4067/ S0718-23762015000100006.

Ferreira L.D.C., Barbosa S.R.C.S., Hoefel J.L. de M., Guimarães R., Floriani D., Tavolaro S.B.F., 2006. Environmental issues, interdisciplinarity, social theory and intellectual production in Latin America, Ambiente \& Sociedade, 9, 2, 9-24.

Gligo N., 2001. La dimensión ambiental en el desarrollo de América Latina, Santiago de Chile, Naciones unidas.

Gonçalves C.W.P., 2001. Geo-grafías. Movimientos sociales, nuevas territorialidades y sustentabilidad, México, Siglo Veintiuno.

Gudynas E., 1999. Concepciones de la naturaleza y desarrollo en América Latina, Persona y Sociedad, 13, 1, 101-125.

Gudynas E., 2011. Buen vivir: germinando alternativas al desarrollo, América Latina en movimiento, 462.

Gudynas E., Acosta A., 2011. El buen vivir o la disolución de la idea del progreso, in Rojas M. (Ed.), La medición del progreso y el bienestar. Propuestas desde América Latina, México, Foro consultivo científico y tecnológico de México, 103-110.

Guha R., Martinez-Alier J., 1997. Varieties of environmentalism: essays North and South, London, Earthscan.

Guimarães R.P., 2003. Tierra de sombras: desafios de la sustentabilidad y del desarrollo territorial y local ante la globalización corporativa, Santiago de Chile, Naciones unidas.

Hassan S.-U., Haddawy P., Zhu J., 2013. A bibliometric study of the world's research activity in sustainable development and its sub-areas using scientific literature, Scientometrics, 99, 2, 549-579, doi: 10.1007/s11192-013-1193-3.

Herrera A.O., Scolnik H.D., Chichilnisky G., Gallopin G.C., Hardoy J.E., Mosovich D., Oteiza E., de Romero Brest G. L., Suárez C.E., Talavera L., 1976. Catastrophe or new society?: a Latin American world model, Ottawa, International Development Research Centre.

Herrera A.O., Scolnik H.D., Chichilnisky G., Gallopin G.C., Hardoy J.E., Mosovich D., Oteiza E., de Romero Brest G. L., Suárez C.E., Talavera L., 2004. ¿Catastrofe o nueva sociedad? Modelo mundial latinoamericano. 30 años después, Ottawa, Centro Internacional de Investigaciones para el Dessarrolo.

Heyd T., 2004. Themes in Latin American environmental ethics: community, resistance and autonomy, Environmental Values, 13, 2, 223-242, doi: 10.3197/0963271041159859.

Heyd T., 2005. Sustainability, culture and ethics: models from Latin America, Ethics, Place \& Environment, 8, 2, 223-234, doi.org/10.1080/13668790500237385.

Hibbard K.A., Crutzen P.J., Lambin E.F., Liverman D.M., Mantua N.J., McNeill J.R., Messerli B., Steffen W., 2007. Group report: decadal-scale interactions of humans and the environment, in Costanza R., Graumlich L.J., Steffen W.L. (Eds), Sustainability or collapse? An integrated history and future of people on earth, Cambridge, MIT Press, 341-375.

Hopwood B., Mellor M., O’Brien G., 2005. Sustainable development: mapping different approaches, Sustainable Development, 13, 1, 38-52, doi: 10.1002/sd.244. 
Huanacuni Mamani F., 2010. Vivir bien/buen vivir. Filosofía, politicas, estrategias y experiencias regionales, La Paz, Instituto Internacional de Integración.

Jacobs M., 1999. Sustainable development: a contested concept, in Dobson A. (Ed.), Fairness and futurity. Essays on environmental sustainability and social justice, Oxford/ New York, Oxford University Press, 21-45.

Kajikawa Y., Ohno J., Takeda Y., Matsushima K., Komiyama H., 2007. Creating an academic landscape of sustainability science: an analysis of the citation network, Sustainability Science, 2, 2, 221-231, doi: 10.1007/s11625-007-0027-8.

Kajikawa Y., Tacoa F., Yamaguchi K., 2014. Sustainability science: the changing landscape of sustainability research, Sustainability Science, 9, 4, 431-438, doi: 10.1007/ s11625-014-0244-x.

Leff E. (Ed.), 1986. Los problemas del conocimiento y la perspectiva ambiental del desarrollo, México, Siglo Veintiuno.

Leff E., 1994. Ecología y capital: racionalidad ambiental, democracia participativa y desarrollo sustentable, México, Siglo Veintiuno.

Leff E., 2004. Racionalidad ambiental. La reapropiación social de la naturaleza, México, Siglo Veintiuno.

Leff E., 2012. Latin American environmental thinking. A heritage of knowledge for sustainability, Environmental Ethics, 34, 4, 431-450.

Leff E., 2015. The power-full distribution of knowledge in political ecology. A view from the south, in Perreault T., Bridge G., McCarthy J. (Eds), The Routledge handbook of political ecology, Abingdon/New York, Routledge.

Lélé S.M., 1991. Sustainable development: a critical review, World Development, 19, 6, 607-621, doi.org/10.1016/ 0305-750X(91)90197-P.

Lélé S.M., 2013. Rethinking sustainable development, Current History, 112, 757, 311-316.

Ma C., Stern D.I., 2006. Environmental and ecological economics: a citation analysis, Ecological Economics, 58, 3, 491-506, doi: 10.1016/j.ecolecon.2005.07.023.

Meadows D.H., Meadows D.L., Randers J., Behrens W.W., 1972. The limits to growth. A report for the Club of Rome's project on the predicament of mankind, New York, Universe Books.

Mesarović M.D., Pestel E., 1974. Mankind at the turning point. The second report to the Club of Rome, New York, Dutton.

Prebisch R., 1950. The economic development of Latin America and its principal problems, New York, United Nations.

Price D.J. de S., 1965. Networks of scientific papers, Science, 149, 3683, 510-515, doi: 10.1126/science.149.3683.510.

Price D.J. de S., Beaver D.D., 1966. Collaboration in an invisible college, American Psychologist, 21, 11, 1011-1018, doi: $10.1037 / \mathrm{h} 0024051$.

Quental N., Lourenço J.M., 2012. References, authors, journals and scientific disciplines underlying the sustainable development literature: a citation analysis, Scientometrics, 90, 2, 361-381, doi: 10.1007/s11192-011-0533-4.

Quental N., Lourenço J.M., Nunes da Silva F., 2011. Sustainable development policy: goals, targets and political cycles, Sustainable Development, 19, 1, 15-29, doi: 10.1002/sd.416.
Rozzi R., 2012. South American environmental philosophy: ancestral Amerindian roots and emergent academic branches, Environmental Ethics, 34, 4, 343-366, doi: 10.5840/ enviroethics201234436.

Sachs I., 1974a. Ambiente y estilos de desarrollo, Comercio Exterior, 24, 4, 360-368.

Sachs I., 1974b. Ecodesarrollo: un aporte a la definición de estilos de desarrollo para América Latina, Estudios Internacionales, 7, 25, 57-77, https://revistaei.uchile.cl/ index.php/REI/article/view/17549.

Sachs W., 1997. Sustainable development, in Redclift M.R., Woodgate G. (Eds), The international handbook of environmental sociology, Cheltenham/Northampton, Edward Elgar, 71-82.

Sachs W., 1999. Planet dialectics: explorations in environment and development, London/New York, Zed Books.

Schubert A., Láng I., 2005. The literature aftermath of the Brundtland report 'Our common future'. A scientometric study based on citations in science and social science journals, Environment, Development and Sustainability, 7, 1, 1-8, doi: 10.1007/s10668-003-0177-5.

Sneddon C., Howarth R.B., Norgaard R.B., 2006. Sustainable development in a post-Brundtland world, Ecological Economics, 57, 2, 253-268, doi: 10.1016/j.ecole con.2005.04.013.

Strong M. and Experts on Development and Environment, 1971. The Founex Report, Paris, Mouton.

Van Opstal M., Hugé J., 2013. Knowledge for sustainable development: a worldviews perspective, Environment, Development and sustainability, 15, 3, 687-709, doi: 10.1007/s10668-012-9401-5.

Vanhulst J., 2015. El laberinto de los discursos del Buen vivir: entre Sumak Kawsay y socialismo del siglo XXI, Polis, Revista Latinoamericana, 14, 40, 233-261.

Vanhulst J., Beling A.E., 2013a. Buen vivir et développement durable: rupture ou continuité ? Écologie \& Politique, 46, 1, 41-54.

Vanhulst J., Beling A.E., 2013b. Buen vivir: la irrupción de América Latina en el campo gravitacional del desarrollo sostenible, Revista Iberoamericana de Economía Ecológica, 21, 15-28.

Vanhulst J., Beling A.E., 2014. Buen vivir: emergent discourse within or beyond sustainable development?, Ecological Economics, 101, 54-63, doi: 10.1016/j.ecolecon.2014.02.017.

Vanhulst J., Beling A.E., 2017. Esquisse pour une généalogie glocale du Buen Vivir, Synergies Chili, 13, 15-25.

Vanhulst J., Zaccai E., 2016. Sustainability in Latin America: an analysis of the academic discursive field, Environmental Development, 20, 68-82, doi: 10.1016/j.envdev.2016.10.005.

Villalba B. (Ed.), 2009. Appropriations du développement durable: émergences, diffusions, traductions, Villeneuved'Ascq, Presses universitaires du Septentrion.

Vieira P.F., Ribeiro M.A., Franco R.M., Cordeiro R.C. (Eds), 1998. Desenvolvimento e meio ambiente no Brasil: a contribuição de Ignacy Sachs, Porto Alegre/Florianópolis, Pallotti/APED.

Vivien F.-D., 2005. Le développement soutenable, Paris, La Découverte. 
Wasserman S., Faust K., 1994. Socialnetwork analysis. Methods and applications, Cambridge, Cambridge University Press.

White H.D., 2011. Scientific and scholarly networks, in Scott J., Carrington P.J. (Eds), The SAGE handbook of social network analysis, Los Angeles/London, SAGE, 271-285.

Zaccai E., 2002. Le développement durable. Dynamique et constitution d'un projet, Bruxelles, P. Lang.

\section{Annexes. Le paysage intellectuel du développement durable en Amérique latine Annexe 1. Échantillon des auteurs latino-américains.}

Abogabir Ximena

Abramovay Ricardo

Acosta Alberto

Alimonda Hector

Aliste Enrique

Angel Maya Augusto

Arabany Ramirez Luz

Azuela de la Cueva Antonio

Barkin David

Bermudez Guerrero Olga María

Boff Leonardo

Boisier Sergio

Borrero José María

Brailovsky Antonio

Brondizio Eduardo

Buarque Cristovam

Bugallo Alicia Irene

Bursztyn Marcel

Carmona Maya Sergio Iván

Carosio Alba

Carrizosa Umaña Julio

Castro Herrera Guillermo

Cavalcanti Clovis

Claude Marcel

Corragio Jose Luis

Da Costa Fereira Leila

Da Veiga José Eli

Davalos Pablo

De Lisio Antonio

Drummond José Augusto

Elizalde Hevia Antonio

Eschenhagen Maria Luisa

Escobar Arturo

Esteva Gustavo

Fergusson Alex

Fernández Soriano Armando

Florez Margarita

Floriani Dimas

Foladori Guillermo

Folchi Mauricio

Gabaldón Berti Arnoldo José

Galano Carlos

Gallopin Gilberto

Garcia Guadilla María Pilar

Garcia Henao Lilibeth

Gligo Nicolo

Gomez Echeverri Luis Fernando
Gomez Giraldo Luis Jair

Gomez-Pompa Arturo

Gonzalez Gaudiano Edgar

Govea Hector

Grueso Libia

Gudynas Eduardo

Guimaraes Roberto

Gutiérrez Nájera Raquel

Gutman Pablo

Hajek Ernst

Herrera Amilcar

Hurtubia Jaime

Kwiatkowska Teresa

Lander Edgardo

Larrain Sara

Leff Enrique

Leis Hector Ricardo

Maihold Gunther

Mansilla Hugo Celso Felipe

Max-Neef Manfred

Meléndez Dobles Silvia

Noguera de EcheverrI Ana Patricia

Padua Jose Augusto

Palacio Castañeda German

Pichs Madruga Ramón

Porto Gonçalves Carlos Walter

Quadri Gabriel

Quiroga Martinez Rayen

Rattner Henrique

Reboratti Carlos

Rodriguez Becerra Manuel

Romero Aravena Hugo

Sabatini Francisco

Sachs Ignacy

Sanchez Buitrago Dairo

Sarukhan José

Sawyer Donald

Schuldt Jurgen

Sejenovich Hector

Sunkel Osvaldo

Tobasura Acuña Isaías

Toledo Victor

Urquidi Victor

Villegas Arenas Guillermo

Viola Eduardo

Wilches-Chaux Gustavo 


\section{Annexe 2. Liste complète des auteurs, documents cités et identifiants.}

\begin{tabular}{|c|c|c|c|}
\hline 1 & Abogabir Ximena & 58 & Da Costa Fereira Leila \\
\hline 2 & Abramovay Ricardo & 59 & Da Veiga Jose Eli \\
\hline 3 & Acosta Alberto & 60 & Daly Herman \\
\hline 4 & Acot Pascal & 61 & Davalos Pablo \\
\hline 5 & Adams William Mark & 62 & De Lisio Antonio \\
\hline 6 & Agrawal Arun & 63 & Deleage Jean-Paul \\
\hline 7 & Alimonda Hector & 64 & Dobson Andrew \\
\hline 8 & Aliste Enrique & 65 & Dorst Jean \\
\hline 9 & Altieri Miguel & 66 & Drummond Jose Augusto \\
\hline 10 & Altvater Elmar & 67 & Dryzek John \\
\hline 11 & Angel Maya Augusto & 68 & Dubos René \\
\hline 12 & Arabany Ramirez Luz & 69 & Dumont René \\
\hline 13 & Ayres Robert & 70 & Duncan Otis Dudley \\
\hline 14 & Azuela de la Cueva Antonio & 71 & Dunlap Riley et Catton William \\
\hline 15 & Barbier Edward B. & 72 & Ehrlich et Ehrlich \\
\hline 16 & Barkin David & 73 & Ekins Paul \\
\hline 17 & Barnett \& Morse & 74 & Elizalde hevia antonio \\
\hline 18 & Barry John & 75 & Eriksson Karl-Erik \\
\hline 19 & Beck Ulrich & 76 & Eschenhagen Maria Luisa \\
\hline 20 & Benton Ted & 77 & Escobar Arturo \\
\hline 21 & Berkes Fikret & 78 & Esteva Gustavo \\
\hline 22 & Bermudez Guerrero Olga Maria & 79 & Factor 10 Club \\
\hline 23 & Biermann Frank & 80 & Fearnside Philip \\
\hline 24 & Biswas Asit K. & 81 & Fergusson Alex \\
\hline 25 & Boff Leonardo & 82 & Fernandez Soriano Armando \\
\hline 26 & Boisier Sergio & 83 & Ferry Luc \\
\hline 27 & Bookchin Murray & 84 & Florez Margarita \\
\hline 28 & Borrero Navia Jose Maria & 85 & Floriani Dimas \\
\hline 29 & Boserup Ester & 86 & Foladori Guillermo \\
\hline 30 & Botkin Daniel & 87 & Folchi Mauricio \\
\hline 31 & Boulding Kenneth E. & 88 & Forsyth Tim \\
\hline 32 & Bourg Dominique & 89 & Foster John Bellamy \\
\hline 33 & Brailovsky Antonio Elio & 90 & Founex (rapport) \\
\hline 34 & Brondizio Eduardo & 91 & Freudenburg William \\
\hline 35 & Brown Lester & 92 & Friends of the Earth \\
\hline 36 & Brundtland report & 93 & Funtowicz Silvio \\
\hline 37 & Bryant Raymond & 94 & Gabaldon Berti Arnoldo Jose \\
\hline 38 & Buarque Cristovam & 95 & Gadgil Madhav \\
\hline 39 & Bugallo Alicia Irene & 96 & Galano Carlos \\
\hline 40 & Burkett Paul & 97 & Gallopin Gilberto \\
\hline 41 & Bursztyn Marcel & 98 & Garcia Guadilla Maria Pilar \\
\hline 42 & Buttel Frederick & 99 & Garcia Henao Lilibeth \\
\hline 43 & Callicott J. Baird & 100 & Georgescu-Roegen Nicholas \\
\hline 44 & Carmona Maya Sergio Ivan & 101 & Gligo Nicolo \\
\hline 45 & Carosio Alba & 102 & Godard Olivier \\
\hline 46 & Carrizosa Umana Julio & 103 & Goldsmith Edward \\
\hline 47 & Carson Rachel & 104 & Gomez Echeverri Luis Fernando \\
\hline 48 & Castro Herrera Guillermo & 105 & Gomez Giraldo Luis Jair \\
\hline 49 & Cavalcanti Clovis & 106 & Gomez-Pompa Arturo \\
\hline 50 & Claude Marcel & 107 & Gonzalez Gaudiano Edgar \\
\hline 51 & Commoner Barry & 108 & Goodland Robert \\
\hline 52 & Conway Gordon Richard & 109 & Gorz André \\
\hline 53 & Coraggio Jose Luis & 110 & Govea Hector \\
\hline 54 & Costanza Robert & 111 & Grinevald Jacques \\
\hline 55 & Cotgrove Stephen & 112 & Grueso Libia \\
\hline 56 & Crosby Alfred & 113 & Grundmann Reiner \\
\hline 57 & Crutzen Paul & 114 & Gudynas Eduardo \\
\hline
\end{tabular}




\section{Annexe 2. (Suite)}

\begin{tabular}{|c|c|c|c|}
\hline 115 & Guha Ramachandra & 177 & Peet Richard \\
\hline 116 & Guimaraes Roberto & 178 & Pichs Madruga Ramon \\
\hline 117 & Gutierrez Najera Raquel & 179 & Pigou Arthur \\
\hline 118 & Gutman Pablo & 180 & Pimentel David \\
\hline 119 & Hajek Ernst & 181 & Pinchot Gifford \\
\hline 120 & Hannigan John & 182 & PNUMA \\
\hline 121 & Hardin Garett & 183 & Porto Goncalves Carlos Walter \\
\hline 122 & Hawley Amos & 184 & Prigogine Ilia \\
\hline 123 & Herrera Amilcar & 185 & Quadri Gabriel \\
\hline 124 & Hurtubia Jaime & 186 & Quiroga Martinez Rayen \\
\hline 125 & Illich Ivan & 187 & Raskin Paul \\
\hline 126 & Ingold Tim & 188 & Rattner Henrique \\
\hline 127 & IPCC & 189 & Ravetz Jérôme \\
\hline 128 & IUCN World Strategy & 190 & Reboratti Carlos \\
\hline 129 & Jackson Tim & 191 & Redclift Michael \\
\hline 130 & Janicke Martin & 192 & Reed David \\
\hline 131 & Jasanoff Sheila & 193 & Rees William \\
\hline 132 & Jollivet Marcel & 194 & Repetto Robert \\
\hline 133 & Jonas Hans & 195 & Riechmann Jorge \\
\hline 134 & Kneese Allen & 196 & Rifkin Jeremy \\
\hline 135 & Korten David & 197 & Rist Gilbert \\
\hline 136 & Kwiatkowska Teresa & 198 & Rodriguez Becerra Manuel \\
\hline 137 & Lander Edgardo & 199 & Romero Aravena Hugo \\
\hline 138 & Larrain Sara & 200 & Ropke Inge \\
\hline 139 & Latouche Serge & 201 & Sabatini Francisco \\
\hline 140 & Leff Enrique & 202 & Sachs Ignacy \\
\hline 141 & Leis Hector Ricardo & 203 & Sanchez Buitrago Dairo \\
\hline 142 & Lele Sharachchandra & 204 & Sarukhan Jose \\
\hline 143 & Leopold Aldo & 205 & Sawyer Donald \\
\hline 144 & Lipietz Alain & 206 & Schnaiberg Alan \\
\hline 145 & Lomborg Bjorn & 207 & Schuldt Jurgen \\
\hline 146 & Lovelock James & 208 & Schumacher Ernst \\
\hline 147 & Lowy Michael & 209 & Sejenovich Hector \\
\hline 148 & Lubchenko Jane & 210 & Sen Amartya \\
\hline 149 & Macintyre Alasdair & 211 & Shiva Vandana \\
\hline 150 & Maihold Gunther & 212 & Simon Julian \\
\hline 151 & Mansilla Hugo Celso Felipe & 213 & Spaargaren Gert \\
\hline 152 & Margulis Lynn & 214 & Strong Maurice \\
\hline 153 & Martinez-Alier Joan & 215 & Sunkel Osvaldo \\
\hline 154 & Max-Neef Manfred & 216 & Tansley Arthur G. \\
\hline 155 & McCormick John & 217 & Theilard de Chardin Pierre \\
\hline 156 & Meadows et al. (rapport) & 218 & Thoreau Henri David \\
\hline 157 & Melendez Dobles Silvia & 219 & Tobasura Acuna Isaias \\
\hline 158 & Mesarovic Mihajlo y Pestel Eduard (rapport) & 220 & Toledo Victor \\
\hline 159 & Morin Edgar & 221 & Toynbee Arnold \\
\hline 160 & Moscovici Serge & 222 & UNEP \\
\hline 161 & Munasinghe Mohan & 223 & Urquidi Victor \\
\hline 162 & Myers Norman & 224 & Vernadsky Vladimir \\
\hline 163 & Naess Arne & 225 & Villegas Arenas Guillermo \\
\hline 164 & Nijkamp Peter & 226 & Viola Eduardo \\
\hline 165 & Noguera de Echeverri Ana Patricia & 227 & Vitousek Peter \\
\hline 166 & Nordhaus William & 228 & Vivien Franck-Dominique \\
\hline 167 & Norgaard Richard & 229 & Wackernagel Matis \\
\hline 168 & O'Connor James & 230 & Ward Barbara \\
\hline 169 & O'Riordan Tim & 231 & What Now? Report \\
\hline 170 & Odum Eugene P. & 232 & Whyte Lynn \\
\hline 171 & Odum Howard T. & 233 & Wilches chaux Gustavo \\
\hline 172 & Ostrom Elinor & 234 & Wilson Edward O. \\
\hline 173 & Padua Jose Augusto & 235 & Woodgate Graham \\
\hline 174 & Palacio Castaneda German & 236 & Worster Donald \\
\hline 175 & Pearce David & 237 & Zaccai Edwin \\
\hline 176 & Peccei Aurelio & & \\
\hline
\end{tabular}

En gris : les auteurs latino-américains.

Citation de l'article : Vanhulst J., Zaccai E., 2019. Le paysage intellectuel du développement durable en Amérique latine. Une analyse de réseau à partir des citations d'auteurs latino-américains. Nat. Sci. Soc. 27, 3, 278-296. 